\title{
Comparative survival analyses among captive chimpanzees (Pan troglodytes) in America and Japan
}

\author{
Judy Che-Castaldo $^{1}$, Kristin Havercamp ${ }^{2}$, Koshiro Watanuki ${ }^{2}$, Tetsuro Matsuzawa ${ }^{3,4}$, Satoshi Hirata ${ }^{\text {Corresp., } 2,5}$, \\ Stephen Ross ${ }^{\text {Corresp. } 6}$ \\ ${ }^{1}$ Alexander Center for Applied Population Biology, Conservation \& Science Department, Lincoln Park Zoo, Chicago, Illinois, United States \\ 2 Wildlife Research Center, Kyoto University, Kyoto, Japan \\ 3 Chubu Gakuin University, Gifu, Japan \\ 4 Division of the Humanities and Social Science, California Institute of Technology, Pasadena, California, United States \\ 5 Kumamoto Sanctuary, Kyoto University, Kumamoto, Japan \\ 6 Lester E. Fisher Center for the Study and Conservation of Apes, Lincoln Park Zoo, Chicago, Illinois, United States \\ Corresponding Authors: Satoshi Hirata, Stephen Ross \\ Email address: hirata.satoshi.8z@kyoto-u.ac.jp, sross@lpzoo.org
}

Detailed, long-term datasets on the life histories of long-lived species such as great apes are necessary to understand their survival patterns but are relatively rare. Such information requires prolonged and consistent record-keeping over many generations, so for chimpanzees (Pan troglodytes), this equates to many decades of input. As life history variables can be altered by differences in environmental influences (whether natural or artificial), there is substantial value to being able to compare across populations. Here, we present the first comparative analysis of life history data for two ex situ chimpanzee populations residing in North America (1975-2020; $n=730$ ) and Japan (1980-2020; $n=660$ ). Overall, survival patterns were similar between regions, and the median life expectancy from birth is estimated at $35.7(95 \% \mathrm{Cl}=32.4-40.0)$ years for females and 30.1 (27.3-34.3) years for males across both populations. Females who survive to their first birthday are estimated to survive 42.4 (40.0-46.3) years and males 35.5 (32.6-38.0) years. We found that birth type (wild-born or captive-born) did not influence survival patterns in either population, but there were differential effects of sex on longevity. In the America population, males had higher mortality rates than females, whereas in the Japan population we found no differences between the sexes. First year mortality did not differ between populations for males (18-20\%), but for females it was lower in America (15\%) compared to Japan (25\%). Survival patterns of chimpanzees in the present study will be useful for future investigation into potential causes of regional differences and crossspecies comparisons. 
1 Comparative survival analyses among captive

2 chimpanzees (Pan troglodytes) in America and Japan

4 Judy P. Che-Castaldo ${ }^{1}$, Kristin Havercamp ${ }^{2}$, Koshiro Watanuki², Tetsuro Matsuzawa ${ }^{3,4}$, 5 Satoshi Hirata ${ }^{2,5}$, Stephen R. Ross 6

$6{ }^{1}$ Alexander Center for Applied Population Biology, Conservation \& Science Department,

7 Lincoln Park Zoo, Chicago, Illinois, United States

$8{ }^{2}$ Wildlife Research Center, Kyoto University, Kyoto, Japan

$9{ }^{3}$ Chubu Gakuin University, Gifu, Japan

$10{ }^{4}$ Division of the Humanities and Social Science, California Institute of Technology, Pasadena, 11 California, United States

$12{ }^{5}$ Kumamoto Sanctuary, Kyoto University, Kumamoto, Japan

$13{ }^{6}$ Lester E. Fisher Center for the Study and Conservation of Apes, Lincoln Park Zoo, Chicago, 14 Illinois, United States

16 Corresponding Authors:

17 Satoshi Hirata ${ }^{1,2}$ and Stephen R. Ross ${ }^{3}$

$18{ }^{1}$ Wildlife Research Center, Kyoto University, Tanaka Sekidencho, Sakyo Ward, Kyoto, 60619 8203, Japan

$20{ }^{2}$ Kumamoto Sanctuary, Kyoto University, Kumamoto, Japan

21 Email address: hirata.satoshi.8z@kyoto-u.ac.jp

$22{ }^{3}$ Lester E. Fisher Center for the Study and Conservation of Apes, Lincoln Park Zoo, $2001 \mathrm{~N}$

23 Clark Street, Chicago, Illinois, 60614, United States

24 Email address: sross@lpzoo.org

25

26

27

28

29

Submission admin:

Kristin Havercamp

kristin.havercamp.24z@st.kyoto-u.ac.jp 
30

31

32

33

34

35

36

37

38

39

40

41

42

43

44

45

46

47

48

49

50

51

52

53

54

55

56

57

58

59

60

61

62

63

64

65

66

\section{Abstract}

Detailed, long-term datasets on the life histories of long-lived species such as great apes are necessary to understand their survival patterns but are relatively rare. Such information requires prolonged and consistent record-keeping over many generations, so for chimpanzees (Pan troglodytes), this equates to many decades of input. As life history variables can be altered by differences in environmental influences (whether natural or artificial), there is substantial value to being able to compare across populations. Here, we present the first comparative analysis of life history data for two ex situ chimpanzee populations residing in North America (1975-2020; $\mathrm{n}=730)$ and Japan $(1980-2020 ; \mathrm{n}=660)$. Overall, survival patterns were similar between regions, and the median life expectancy from birth is estimated at $35.7(95 \% \mathrm{CI}=32.4-40.0)$ years for females and 30.1 (27.3-34.3) years for males across both populations. Females who survive to their first birthday are estimated to survive 42.4 (40.0-46.3) years and males 35.5 (32.6-38.0) years. We found that birth type (wild-born or captive-born) did not influence survival patterns in either population, but there were differential effects of sex on longevity. In the America population, males had higher mortality rates than females, whereas in the Japan population we found no differences between the sexes. First year mortality did not differ between populations for males (18-20\%), but for females it was lower in America (15\%) compared to Japan (25\%). Survival patterns of chimpanzees in the present study will be useful for future investigation into potential causes of regional differences and cross-species comparisons.

\section{Introduction}

Studying life history patterns of non-human primates is important for understanding the evolution of human life histories and senescence (Bronikowski et al., 2011). In particular, chimpanzee (Pan troglodytes) survival and mortality patterns have been studied to explore differences with other species, among populations of chimpanzees in the wild, and between wild and captive populations (e.g. Earnhardt et al., 2003; Hill et al., 2001; Thompson et al., 2007; Muller \& Wrangham, 2014; Tidière et al., 2016; Wood et al., 2017; Davison \& Gurven, 2021). Detailed, long-term datasets on life histories are rare but important for capturing survival rates at later ages, especially for long-lived species such as chimpanzees. Comparing chimpanzee survival across different captive populations may expose similarities or differences in survival patterns which could be further examined for determining best management strategies such as husbandry techniques, diet and veterinary care.

Chimpanzees are one of humans' closest living relatives and have been studied for decades, which has allowed for the comparison of life history patterns to shed light on human evolution (e.g. Hawkes, Smith \& Robson, 2009; Hill et al., 2001; Muller \& Wrangham, 2014; Thompson et al., 2007; Wood et al., 2017; Davison \& Gurven, 2021). Life expectancy at birth for huntergatherers ranges from $21-37$ years across groups and between $26-43 \%$ of people survive to age 
6745 (Gurven \& Kaplan, 2007), similar to what has been found in a wild (Wood et al., 2017) and a

68

69

70

71

72

73

74

75

76

77

78

79

80

81

82

83

84

85

86

87

88

89

90

91

92

93

94

95

96

97

98

99

100

101

102

103

104

105

106 captive chimpanzee population (Havercamp et al., 2019). The average life expectancy of wild chimpanzees varies across populations (Table 1). Data from five sites across Africa show that individuals who survive to adulthood ( $14 \mathrm{y}$ ) have a life expectancy of approximately 29 years (Hill et al., 2001). In Kanyawara in East Africa, adult chimpanzees (14 y) can expect to live 38 years (Muller \& Wrangham, 2014) and at Ngogo, also in East Africa, 43 years (Wood et al., 2017). In captivity in Japan, a 14-year old individual is expected to reach 42 years of age (Havercamp et al., 2019). Longevity estimates of chimpanzees from birth are lower due to the high risk infant period, from 13 years across five sites (Hill et al., 2001), 19 years at Kanyawara (Muller \& Wrangham, 2014), 28 years in captivity (Havercamp et al., 2019), to 33 years at Ngogo (Wood et al., 2017). Typically, mammals live longer in captive environments such as zoos compared to in the wild, although some long-lived species such as elephants and chimpanzees do not (Tidière et al., 2016).

There are several existing reports on captive chimpanzee survival which serve to inform our understanding of life histories (Table 1). Unfortunately, they are now outdated, derived from relatively small samples or missing data on older individuals. Courtenay \& Santow (1989) published the first life table, though it was derived from just 87 individuals and mortality estimates were only calculated up to age 30 due to the short history of the population at that time. Dyke et al. (1995) presented modelled data including 1,488 individuals from three colonies in America and reported that males and females had an expected lifespan of 21 years and 29 years respectively when calculated from birth. When calculated from adulthood (14 years), males had an expected lifespan of 33 years and females 41 years. However, because the modelled life tables represented an average of various sources of variability, they urged caution in using these data as a representation of chimpanzees. In a later study, chimpanzees at Taronga Park Zoo in Australia experienced higher mortality risk from birth than wild chimpanzees in Mahale or Gombe. They also reported that females had greater life expectancy than males, however the size of the captive population was only 113 and the life table reached only 51 years of age (Littleton, 2005).

Historically, America and Japan have held the largest number of captive chimpanzees in the world, over 3000 individuals combined, and the trajectory of those populations have been described in detail by Hirata et al. (2020). The earliest record of a chimpanzee in America is from 1902, whereas the first chimpanzee arrived in Japan around two decades later, in 1921. America adopted the Convention on International Trade in Endangered Species of Wild Fauna and Flora (CITES) in 1975, ceasing the importation of chimpanzees captured in Africa. Japan followed soon after ratifying CITES in 1980, however was still able to import wild chimpanzees under special circumstances for research until 1983. Broadly speaking, the populations are relatively similar in terms of their management at an individual and group level, however there are likely some differences at the population level. While the Japan population is largely selfcontained and its growth is dependent on births in zoos, the America population has a significant

Peer) reviewing PDF | (2021:03:59294:1:0:NEW 16 Jun 2021) 
107 proportion of chimpanzees that have entered the population from external sources such as

108

109

110

111

112

113

114

115

116

117

118

119

120

121

122

123

124

125

126

127

128

129

130

131

132

133

134

135

136

137

138

139

140

141

142

143

research centers, the entertainment industry, and the pet trade (Hirata et al., 2020).

We synthesize data from these two regional captive populations to yield the largest dataset on chimpanzee life history and report the first systematic comparison of survival patterns for a primate species across regions. We present life tables by age and sex and statistics for chimpanzee survival and life expectancy for both the America population living in zoos accredited by the Association of Zoos and Aquariums (AZA) and the Japan population living in zoos and one sanctuary. We use studbook records, covering the modern history of these apes in each country to examine whether differences in longevity and survival exist between the two populations over a comparable time period. We hypothesized that the two populations would show similar life history patterns for survivorship and longevity, and demonstrate similar influences of sex and birth type (wild or captive born) on these variables. We did not have a priori predictions on how the survival patterns may differ between regions based on our knowledge of the welfare and management of the two populations. Factors contributing to survival rates such as diet, the ways and levels of veterinary care, and the specific physical and social environments differ between regions but also vary greatly between facilities within each region. Thus there is unlikely to be a consistent difference between countries that would impact survival metrics at the regional scale. We also predicted that survivorship for both populations would increase in the more recent timeframe (2001-2020) and tested whether mortality varied by season in the AZA population as was shown in the Japan population (Havercamp et al. 2019). Such cross-region population comparisons may help elucidate potential life history outcomes affected by differences in captive care and management.

\section{Materials \& Methods}

\section{Data collection}

We accessed demographic data for the chimpanzee population housed in AZA accredited facilities via the North American Regional Studbook for Chimpanzees (Ross, 2020), the most robust and consistent record of chimpanzees in the United States. Over more than a century, thousands of chimpanzees have lived in a range of captive settings in America including laboratories, zoos (accredited and unaccredited), sanctuaries (accredited and unaccredited), and in private hands (as pets or performers). Due to the varied nature of housing facilities and the historically loose regulation of cross-facility transfers, there was no comprehensive record of the U.S.-based chimpanzee population until Lincoln Park Zoo's Project ChimpCARE, founded in 2007, began censusing all chimpanzees in the country (www.chimpcare.org). The North American Regional Studbook (Ross, 2020) contains records of 1302 chimpanzees since 1902, most of whom have lived in accredited zoos. At the time of writing, 260 chimpanzees lived in 31 AZA-accredited facilities in the United States, whereas Project ChimpCARE estimates that 1382

PeerJ reviewing PDF | (2021:03:59294:1:0:NEW 16 Jun 2021) 
144 were alive across all settings (including zoos, sanctuaries, private owners, laboratories, and

145

146

147

148

149

150

151

152

153

154

155

156

157

158

159

160

161

162

163

164

165

166

167

168

169

170

171

172

173

174

175

176

177

178

179

180

181

182

183

unaccredited facilities).

Similarly, we accessed data on captive chimpanzees living in zoos and a sanctuary in Japan via the open-access Great Ape Information Network (GAIN; https://shigen.nig.ac.jp/gain/; Havercamp et al., 2019) using PopLink software (Faust et al., 2019). Chimpanzees in Japan have been tracked via GAIN since the program was initiated in 2002 (Matsuzawa, 2016; Ochiai et al., 2015; Watanuki et al., 2014). The database has been utilized for a recent demographic analysis of this population (Havercamp et al., 2019). There are records for 1025 (possibly 1067) individuals who have lived in Japan since 1921, most of whom were housed in zoos, but also in eight biomedical research institutions, universities, and a sanctuary. In total, 205 were subjects of biomedical research (Hirata et al., 2020). At the time of writing, 302 chimpanzees live across 48 facilities including 46 zoos, animal or amusement parks, one university institute (12 individuals; Primate Research Institute of Kyoto University), and one sanctuary (50 individuals; Kumamoto Sanctuary of Kyoto University established in 2011, which was a former biomedical institution before it was reformed into Chimpanzee Sanctuary Uto in 2007; Morimura et al., 2011).

For both populations, we included for analysis individuals with a known birth location who had at least a portion of their lifespan within a time frame that reflected modern population management (1/1/1975 to 2/1/2020 for AZA, 1/1/1980 to 2/1/2020 for Japan). We selected these ranges based on the years when America and Japan ratified CITES. For the AZA population, we also filtered to include the portion of lifespans that occurred within AZA-accredited facilities. We did not include records for chimpanzees who do not live in accredited zoo settings (i.e., laboratory, private, sanctuary). Some individuals had uncertainty in their birth dates, and our results should be interpreted with these in mind. Of the 730 total individuals ( 320 males, 389 females, and 21 individuals of unknown sex) across 94 facilities included from the AZA population, 563 had known birthdates. The remaining had a recorded birth date plus a time window reflecting the recorder's uncertainty in the actual birth date (e.g., Jan. 1, $1993 \pm$ one month): three individuals had estimates that were within four years of the actual birth date, 104 within two years, 28 within one year, one within six months, and 31 within one month. Of the 660 total individuals (292 males, 364 females, 4 individuals of unknown sex) across 77 facilities included from the Japan population, 486 had known birth dates, while 172 had birth date estimates that were within one year and two had estimates within one month of the actual birthdate. Finally, we excluded miscarriage and stillbirth events in the studbook (records of individuals who died on their birthdates), of which there were 59 in AZA and 50 in Japan.

$\underline{\text { Life tables }}$

Using these data, we calculated life tables for each population using PMx software (Ballou, Lacy $\&$ Pollak, 2020), consisting of Kaplan-Meier estimates of annual age-specific mortality $\left(q_{x}\right)$ and fecundity $\left(M_{x}\right)$ rates (Lacy, Ballou \& Pollak, 2012). We included individuals of unknown sex, all

Peer) reviewing PDF | (2021:03:59294:1:0:NEW 16 Jun 2021) 
184 of whom died (4 individuals in Japan and 19 in AZA) or were lost to follow-up ( 2 in AZA)

185

186

187

188

189

190

191

192

193

194

195

196

197

198

199

200

201

202

203

204

205

206

207

208

209

210

211

212

213

214

215

216

217

218

219

220

221

222

223 within their first year of life, in the life table calculations to more accurately reflect the observed rate of first-year mortality. They were distributed as 0.5 male and 0.5 female following the standard in PMx (indivdiuals of unknown sex were excluded from all remaining analyses). To compare against a recently published life table for a wild chimpanzee population in Gombe, Tanzania (Bronikowski et al., 2016), we calculated $l_{x}$ (survival to age $\mathrm{x}$ ) using $q_{x}$ (probability of death within a year for age $\mathrm{x}$ individuals) from our PMx life tables as: $l_{x}=l_{x-1} *\left(1-q_{x-1}\right)$, and $l_{o}=$ 1.0 .

\section{Survival patterns and population comparisons}

We conducted survival analyses (Kleinbaum \& Klein, 2005) using the survival R package (Therneau, 2021) to describe the survival patterns in each population and to test for differences between the two populations. First, we used Cox proportional hazards regression to test whether survival curves differed by sex, birth type (wild-born or captive-born), and their interaction for each population separately. After excluding individuals of unknown sex, there were 69 wild-born males, 251 captive-born males, 100 wild-born females, and 289 captive-born females in the AZA population. For the Japan population, there were 62 wild-born males, 230 captive-born males, 105 wild-born females, and 259 captive-born females. We then combined the data for both populations and tested whether survival curves differed by sex, birth type, region (AZA or Japan), and all possible interactions. We removed any non-significant ( $\mathrm{p}>0.05$, but all had $\mathrm{p}>0.1)$ interaction terms in the final models. We also used the Kaplan-Meier method to fit observed survival curves based on the significant predictors, and to estimate the median life expectancy for each population by sex. We performed survival analyses starting at birth and starting at age one to assess whether patterns change when neonatal mortality was excluded.

Distribution of age at death by population

We next examined longevity over time in each population. We first calculated the age at mortality for each observed death event and divided the events into two time periods ("early" = 1975-2000 for AZA and 1980-2000 for Japan, "recent" = 2001-2020 for both). In total, there were 197 deaths observed in the early period and 150 in the recent period for the AZA population, and 139 deaths observed in the early period and 168 in the recent period for the Japan population. We then used Poisson regression to test whether the mean age at mortality differed based on sex, birth type, and between the two time periods. That is, we modeled the observed ages at mortality as a Poisson-distributed response variable in a generalized linear model with a log link function to analyze the effects of sex, birth type, and time period on the mean age at death. We used the function $g l m$ in the stats R package (R Core Team, 2020), and specified the quasipoisson family to account for overdispersion in the response. Because no deaths before age one were observed in wild born individuals, we conducted the analysis using only mortalities that occurred on or after the first birthday.

Peer) reviewing PDF | (2021:03:59294:1:0:NEW 16 Jun 2021) 
224 Seasonal death patterns

225 Finally, we assessed seasonal patterns in mortality for the AZA population, replicating an 226 analysis previously conducted for the Japan population (Havercamp et al., 2019). For this, we 227 tallied the number of observed deaths by season (spring = March through May, summer $=$ June 228 through August, autumn = September through November, winter $=$ December through February). 229 We applied a Chi-square goodness of fit test (using function chisq.test in the stats R package [R 230 Core Team, 2020]) to compare the observed proportion of mortality in each season to the 231 expected proportion ( $25 \%$ each, or evenly distributed across seasons). We also analyzed only mortalities that occurred on or after the first birthday, and re-analyzed data from the Japan population to match the time frame used in this study (1980-2020). Except where specified, all survival and statistical analyses were performed in R (R Core Team, 2020) and statistical significance was determined using the criterion $\mathrm{p} \leq 0.05$.

\section{Results}

\section{Life tables}

238

239

240

241

242

243

244

245

246

247

248

249

250

251

252

253

254

255

256

257

258

259

260

261

We present the full life table for the AZA population and Japan population (Tables S1 and S2, respectively; Fig. 1). The longest observed lifespan was longer in the AZA population, with one female surviving to an estimated age of 79 , whereas one male survived to estimated age 68 in the Japan population. The longest-lived captive-born (confirmed age) individual in AZA was 57 and in Japan is 53 (still alive). First year mortality was significantly lower in AZA (15\%) than Japan $(25 \% ; z=-2.3, p=0.02)$ for females, and similar between AZA (18\%) and Japan (20\%) for males $(z=-0.6, p=0.58)$. Comparing the survival curves for our two captive populations against that for a wild population from Tanzania, we found that captive chimpanzees experienced a higher survival probability than wild chimpanzees starting at age 2 (Fig. 1).

\section{Survival patterns and population comparisons}

When analyzing each population separately, we found survival patterns differed by sex but not birth type in the AZA population. AZA males had higher overall mortality rates than AZA females, both when examining survival from birth (coefficient $=0.41, \mathrm{z}=2.7, P<0.01$ ) and from age one (coefficient $=0.51, \mathrm{z}=2.8, P<0.01$ ). Survival patterns did not differ by sex or birth type in the Japan population ( $P>0.15$ in all cases). Accordingly, median life expectancy estimates from birth are lower for males than females in AZA (28.4 years and 36.7 years, respectively) but similar between sexes in Japan (34.0 years and 34.1 years, respectively; Table 2). From age one, a similar difference in median life expectancy exists between the sexes in AZA (32.5 years for males, 41.9 years for females), and a slight difference appears in Japan (37.7 years for males, 42.4 years for females; Table 2).

When combining data from both populations to compare survival patterns between regions, the results differed slightly depending on whether we included or excluded neonatal mortality (Fig. 
262 S1). Survival patterns from birth showed a significant effect of $\operatorname{sex}$ (coefficient $=0.39, \mathrm{z}=3.2, P$

$263<0.01$ ) and an interaction between region and sex (coefficient $=-0.39, \mathrm{z}=-2.3, P=0.02$ ): males

264 have lower survival than females overall, but the difference is larger in AZA than Japan.

265 Survival from age one showed an effect of $\operatorname{sex}$ (coefficient $=0.31, \mathrm{z}=3.0, P<0.01$ ), again with

266 higher mortality for males. The Japan population had slightly lower mortality rates than the AZA

267 population, but the difference was not significant (coefficient $=-0.19, \mathrm{z}=-1.8, P=0.07$; Fig. S1)

268 and there were no significant interactions among predictors.

269

270

Distribution of age at death by population

271 Comparing longevity over time also showed slight differences between populations, with

272 interacting effects of sex, birth type, and time period on age at death for both populations (Table

273 3; Fig. 2). Although we did not analyze deaths before age one in this analysis, we note that more

274 neonatal deaths occurred before 2001 than since 2001 in captive-born individuals; neonatal

275 deaths were not possible to observe in wild born individuals. For the AZA population, 43 of 141

276 observed deaths (30\%) in the early time frame occurred before the first birthday, compared to 15

277 of $139(11 \%)$ deaths observed in the recent time frame having occurred before age one. For the

278 Japan population, 45 of 111 deaths (41\%) in the early time frame occurred before the first

279 birthday, compared to 43 of 146 deaths $(29 \%)$ in the recent time frame.

280

281 For the AZA population, the mean ages at death differed by time period and birth type but not by

282 sex (Table 3), with mortality on average occurring later for wild born individuals and in the

283 recent time frame (Fig. 2A). The two-way interaction between time period and birth type was

284 also significant (Table 3), with a slightly larger difference in age at death between captive and

285 wild born individuals in the later time frame compared to the earlier time frame (Fig. 2). When

286 focusing specifically on the early time period for the AZA population, we found the median age

287 of death was very similar for wild-born males and females ( $\mathrm{F}=26.4, \mathrm{M}=27.8)$ and lower for

288 captive-born individuals $(\mathrm{F}=11.1, \mathrm{M}=10.3)$. Considering only the more recent time frame, the

289 mean age at death was higher for both wild-born $(\mathrm{F}=47.0, \mathrm{M}=42.0)$ and captive born $(\mathrm{F}=27.1$,

$290 \mathrm{M}=21.3$ ) chimpanzees. It is important to note that the population was younger overall in the early

291 time period: the mean age of the AZA population was only 14.5 in the early time period

292 (averaged across years within the time frame), and 23.7 in the recent time period (see Fig. 3 for

293 mean age of the population in each year).

294

295 For the Japan population, the mean ages at death differed by all predictors (Table 3), and the

296 three-way interaction as well as the two-way interaction between sex and birth type were also

297 significant (Table 3). The three-way interaction meant that, for example, the difference in age at

298 death between males and females depended on both the time period and birth type. Captive born

299 females died at a younger age than captive born males, but wild born females survived to an

300 older age than wild born males, and this was more apparent in the early time period. The

301 estimated mean age at death in the early time period was 22.9 and 17.5 for wild born females and 
302 males, respectively, and 11.3 and 17.8 for captive females and males, respectively. In the recent 303 time period, the estimated mean age at death was 38.7 and 38.4 for wild born females and males, 304 respectively, and 22.2 and 22.9 for captive females and males, respectively. For context, the 305 mean age of the Japan population was 11.1 in the early time period and 22.2 in the recent time 306 period (Fig. 3).

307

308

309

310

311

312

313

Seasonal death patterns

In examining seasonal patterns of mortality, we confirmed previously published findings (Havercamp et al. 2019) that deaths in the Japan population were not evenly distributed across seasons. To compare to these earlier reports, we used only data since 1980 and found a significant deviation from the expectation of even distribution both when considering all deaths 314 0.01), with the largest proportion of deaths occurring in winter. We did not find such a seasonal 315 pattern in the AZA population, both in analysis of all deaths $\left(\chi^{2}=6.37, \mathrm{df}=3, P=0.09\right)$ and 316 when considering only deaths after age one $\left(\chi^{2}=4.20, \mathrm{df}=3, P=0.24\right.$; Fig. 4$)$.

\section{Discussion}

318 Although there are several published reports on life histories of captive chimpanzee populations 319 (Courtenay \& Santow, 1989; Dyke et al., 1995; Littleton, 2005; Havercamp et al., 2019), we 320 believe this is the first to compare the life histories of two large captive chimpanzee populations 321 from different regions. Such a comparative approach provides additional insights that may 322 inform managers about the life history characteristics that are more (or less) flexible or 323 potentially affected by different management practices.

324

325

326

327

328

329

330

331

332

333

334

335

336

337

338

339

Both of these populations of chimpanzees have been evaluated in previous studies, but the current datasets differ from previous ones in some important ways, primarily to facilitate crossregion comparisons. Earnhardt et al. (2003) used data from the AZA studbook to compare life histories of these chimpanzees to wild chimpanzees living at Gombe Stream National Park in Tanzania. That dataset represented 524 zoo-housed chimpanzees living from 1963 to 2003, compared to 730 from 1975 to 2020 in the current analysis. Likewise, an earlier study (Havercamp et al., 2019) analyzed the historical chimpanzee population living in Japan, using data for 821 chimpanzees from 1921 to 2018 compared to 660 from 1980 to 2020. For both populations, the current analysis represents a more narrow and more recent time frame, and the median life expectancies from the previous analyses were lower than those derived from this study, especially for females. This may be attributed in part to the more recent time frame reflecting more modern management practices that positively influence life history outcomes for these managed populations. For the AZA population, the earlier analysis (Earnhardt et al., 2003) included data up to 2003 and thus the younger population at that time (Fig. 3) could have also contributed to the shorter life expectancy estimates. However, the earlier analysis of the Japan 
340 population (Havercamp et al., 2019) included data through 2018 so the difference in our analysis 341 is mainly excluding the earlier records, and thus are more likely to reflect recent refinements in

342 management.

343

344 Combined, the dataset used here represents the lives of 1390 chimpanzees living in accredited 345 zoos in America and zoos and a sanctuary in Japan. Using data from birth, median life

346

347

348

349

350

351

352

353

354

355

356

357

358

359

360

361

362

363

364

365

366

367

368

369

370

371

372

373

374

375

376

377

378 expectancy (MLE) is 35.7 for females and 30.1 for males (Table 2), which is higher than most longevity estimates for wild chimpanzees. Hill et al. (2001) reported an MLE of just 13 years from data collected at five sites across Africa (see Table 1), and Muller \& Wrangham (2014) reported an MLE of 19 years at Kanyawara, Uganda. However, recent data from Ngogo, Uganda placed life expectancy at over 33 years for that population (Wood et al., 2017). In a direct comparison of wild and captive chimpanzee survivorship, Earnhardt et al. (2003) found that chimpanzees from AZA zoos typically lived longer than those living in Gombe Stream National Park in Tanzania. In comparing the survival curves of our two captive populations with a wild chimpanzee population living in Tanzania (Bronikowski et al., 2016; Fig. 1), we also found support for this pattern; at almost all ages from birth and from one year of age, captive chimpanzees experienced a higher survival probability. Interestingly, although Tidière et al. (2016) showed that mammals generally live longer in captive environments such as zoos compared to in the wild, they found the opposite trend for chimpanzees specifically. Moreover, survival statistics can vary greatly across wild populations (Davison \& Gurven, 2021), so our comparison does not represent all wild populations (i.e., see Wood et al. 2017 for a population with higher survivorship). While these comparisons are worthwhile, we must acknowledge that survivorship comparisons are complicated because of the difficulty in assessing early life mortality for wild chimpanzees, including stillbirths. We removed stillbirths from these analyses, but it is impossible to say how stillbirth rates in captive settings, documented at $12 \%$ for AZA (Saiyed et al., 2018) and 15\% for Japan (Havercamp et al., 2019), compare to those experienced by wild-living chimpanzees.

One of the most robust findings in this analysis was the sex difference in mortality demonstrated in the AZA chimpanzee population. Whether calculated from birth or after the age of one, males had higher mortality rates and shorter life expectancies when compared to females in the same managed population. Such sex differences are reflected by findings from a number of wild chimpanzee populations (Hill et al., 2001; Muller \& Wrangham, 2014; Wood et al., 2017) as well as other mammals (Tidière et al., 2016) and are broadly consistent with sexual selection theory. For many species, including chimpanzees, males are perceived as engaging in more risky behaviors (for instance, hunting and intergroup aggression) which increase the mortality risk. The human literature also reports female advantages in life expectancy that could be tied to a range of genetic, hormonal, metabolic, immune function and other biological factors (Seifarth et al., 2012). For instance, Vina et al. (2011) showed that the higher levels of estrogens in human 
379 females buffer them against the negative effects of aging, by up-regulating the expression of

380

381

382

383

384

385

386

387

388

389

390

391

392

393

394

395

396

397

398

399

400

401

402

403

404

405

406

407

408

409

410

411

412

413

414

415

416

417

418

antioxidant and longevity-related genes.

Interestingly, such sex differences were not evident in the Japanese population and may be explained in part by regional differences in early life histories. Female first-year mortality in Japan was significantly higher than in AZA, suggesting that challenges in birth management and early life care may contribute to increased female mortality rates and a narrowing of the sex gap in mortality one might expect. Comparing the two regions, while female survival seems similar for females in both regions, males in the AZA population have lower survival than males in the Japan population. Explanations for these differences are unclear to us at this time. Though housing conditions and group compositions are not recorded as part of these datasets, we know anecdotally that in general, chimpanzee groups in AZA are larger than those in Japanese zoos and are more likely to have a multimale composition. Historically, some have speculated that males living with fewer or no other males may be under lower daily stress (Alford et al., 1995; Williams et al., 2010), but recent studies contradict this idea and demonstrate that males can live together in mixed-sex or multi-male groups without experiencing heightened aggression or stress levels (Neal Webb, Hau \& Schapiro, 2019; Ross et al., 2009; Seres, Aureli \& de Waal, 2001; Yamanashi et al., 2016). In a multi-institutional study of AZA-accredited zoos, multi-male groups showed lower rates of wounding than single male groups (Ross et al., 2009). Likewise, an all-male group did not show heightened hair cortisol (HC) concentration (a proxy for stress), whereas the alpha male in a mixed-sex group showed the highest level of $\mathrm{HC}$ and aggression (Yamanashi et al., 2016). Nonetheless, the effect of social group composition on health and stress levels is not clear, and so whether this may create a difference in male survival between the two regions is unknown. Other potential factors, such as diet differences in the two regions, may be particularly ripe for future study and we encourage further refinement and extension of cross-regional comparisons of life histories for chimpanzees and other species under intense population management.

In addition to sex effects on life expectancy, we examined the effects of whether chimpanzees were born in the wild or born in captivity. Overall survival patterns did not differ for those born in captive settings compared to those born in the wild, for either the AZA or the Japan population. However, differences in age at death between sexes depended on birth type. In the AZA population, males died younger than females, but this sex difference was smaller for wildborn versus captive born individuals. In Japan, captive-born males tended to die later than captive-born females, but wild-born males died younger than wild-born females. These analyses of birth origin need to be interpreted in context of the fact that early life histories of wild-born individuals are not available (i.e. there are fewer observed deaths in the younger ages, before transfer into captive populations). Also, many wild-born individuals who were captured may not have survived the travails of capture and transport and are therefore not represented in these analyses. This may be an example of the related concepts of selective disappearance and

Peer] reviewing PDF | (2021:03:59294:1:0:NEW 16 Jun 2021) 
419 mortality selection (Vaupel et al., 1979; Vaupel \& Yashin, 1985; Hämäläinen et al., 2014) in 420 which the capture process could have selected for healthier individuals who would thus have 421 higher survival going forward. This may also explain why we did not see the effect of birth type 422 in the survival analyses, which take into account which portions of the lifespan are observed and 423 therefore provide more robust results. As such, we urge caution against over-interpretation of the 424 results that may suggest that wild-born chimpanzees have some form of extra resilience that

425

426

427

428

429

430

431

432

433

434

435

436

437

438

439

440

441

442

443

444

445

446

447

448

449

450

451

452

453

454

455

456

457

458 boosts their longevity.

We also examined how life history characteristics may have changed over time by comparing data from an early period (1975/1980-2000) to a later period (2001-2020). In both regions, age at death has increased in the most recent 20-year period compared to the two decades prior. However, it is important to note that this only represents deaths that had occurred in these periods and because the population was younger overall in the early period, the average age at death was also younger. For example, it does not mean that a captive-born AZA male living in the early time period would only live to 10; rather that captive-born AZA males that died in the early time period were on average aged 10. The median age of both populations is increasing over time (Fig. 3) and will likely continue to increase as more animals have the chance to live out their lifespan. These results demonstrate that focusing only on ages at death can be misleading for determining animal life expectancy, similar to an earlier study on captive elephants (Wiese \& Willis, 2004). This artefact also makes it difficult to test whether there are differences in survival over time due to advancements in care and management protocols as well as veterinary capabilities over the past forty years. In the AZA population for instance, there have been significant improvements in a number of aspects of behavioral management including the promotion of larger and more natural (e.g. multimale) groups and the integration of fissionfusion management systems that may promote a more natural social setting for zoo-housed chimpanzees. Likewise, veterinary expertise surrounding sedation protocols (Naples, Langan \& Kearns, 2010), diet (Struck et al., 2007) and wound management (Baker et al., 2000) may contribute to improving health, welfare and potentially longevity in recent years. Similar activities are being done in Japan as well to promote environmental enrichment (Morimura \& Ueno, 1999; Morimura, 2003), stress monitoring (Yamanashi et al., 2013), method of sedation (Miyabe-Nishiwaki et al., 2021), and detection of zoonotic pathogens (Kooriyama et al., 2013).

Finally, we validated the findings of Havercamp et al. (2019) showing that chimpanzees in the Japan population were more likely to die in winter months. For the AZA population, our data suggest that deaths may peak in both the winter and summer periods (compared to spring and autumn; Fig. 4) but this seasonal pattern was not statistically significant. Although Japan is geographically much smaller than America, both countries are characterized by a broad degree of climatic variation. We are unable to specify if such impacts are particularly prevalent in regions which experience particularly cold winters, though further detailed study of these climate-related effects would be welcome. Nonetheless, these findings provide further support that husbandry 
459 efforts should be intensified during the winter season (e.g. continuous availability of temperature 460 controlled rooms, special diet, warming enrichment, additional health monitoring), especially 461 towards infants, as chimpanzees are likely not well adapted to cold weather and its effects on 462 health. The challenges of managing chimpanzees, who have evolved in equatorial climates, in 463 winter times remains an important consideration.

\section{Conclusions}

465 We compiled the largest dataset on chimpanzee life history, consisting of nearly 1400 individuals 466 and spanning four decades, and compared life history patterns in captive chimpanzee populations 467 from two different regions. Despite some differences in management practices, the overall life 468 expectancy and survival patterns were similar between the two regions. However, survivorship 469 was lower for males than for females in the AZA population but similar among sexes in the 470 Japan population. The Kaplan-Meier median life expectancy from birth was 35.7 (32.4-40.0) 471 years for females and 30.1 (27.3-34.3) years for males across both populations, which is higher 472 than most longevity estimates reported for wild chimpanzees. Birth type (wild-born or captive473 born) did not influence survival patterns in either population, and the seasonal death pattern 474 previously shown in the Japan population was not found in the AZA population. We were unable 475 to fully assess changes in survival over time due to the long lifespan of the species and many 476 individuals having yet to live out their full lives. Moreover, our current estimates of median life 477 expectancy will likely increase in future investigations as animals continue to age in both 478 populations. The opportunity to quantitatively compare life history patterns between large 479 populations of managed species is relatively rare, especially with large and long-lived animals 480 such as chimpanzees. Further investigation will increase the potential of these data to inform 481 important population management strategies.

\section{Acknowledgements}

483 We thank Lisa Faust and Kristine Schad Eebes for helpful discussions regarding studbook 484 analyses. We are also grateful to Yasuhiro Yoshikawa, Toshikazu Hasegawa, Gen'ichi Idani and 485 all members of the Great Ape Information Network for their support in data collection in Japan. 


\section{References}

488 Alford PL, Bloomsmith MA, Keeling ME, Beck TF. 1995. Wounding aggression during the

489 formation and maintenance of captive, multimale chimpanzee groups. Zoo Biology 14:347-359

490 DOI: 10.1002/zoo.1430140406.

491

492 Baker KC, Seres M, Aureli F, de Waal FB. 2000. Injury risks among chimpanzees in three

493 housing conditions. American Journal of Primatology 51:161-175 DOI: 10.1002/1098-

494 2345(200007)51:3<161::AID-AJP1>3.0.CO;2-5.

495

496

497

498

Ballou JD, Lacy RC, Pollak JP. 2020. PMx: Software for demographic and genetic analysis and management of pedigreed populations (Version 1.6.2.20200110). Chicago Zoological Society,

499

500 Brookfield, IL, USA.

501

Bronikowski AM, Altmann J, Brockman DK, Cords M, Fedigan LM, Pusey A, Stoinski T,

502 Morris WF, Strier KB, Alberts SC. 2011. Aging in the natural world: comparative data reveal similar mortality patterns across primates. Science 331:1325-1328 DOI:

503 10.1126/science.1201571.

504

505

Bronikowski AM, Cords M, Alberts SC, Altmann J, Brockman DK, Fedigan LM, Pusey A,

506 Stoinski T, Strier KB, Morris WF. 2016. Female and male life tables for seven wild primate

507 species. Scientific Data 3:1-8 DOI: 10.1038/sdata.2016.6.

508

509

Courtenay J, Santow G. 1989. Mortality of wild and captive chimpanzees. Folia Primatologica

510 52:167-177 DOI: 10.1159/000156395.

511

512

Davison RJ, Gurven MD. 2021. Human uniqueness? Life history diversity among small-scale

513 societies and chimpanzees. PLoS ONE 16:e0239170 DOI: 10.1371/journal.pone.0239170.

514

515

Dyke B, Gage TB, Alford PL, Swenson B, Williams-Blangero S. 1995. Model life table for

516

517 captive chimpanzees. American Journal of Primatology 37:25-37 DOI: 10.1002/ajp.1350370104.

518 Earnhardt JM, Ross SR, Lonsdorf EV, Pusey AE. 2003. A demographic comparison of wild 519 chimpanzees from Gombe and a managed population from North American zoos. American 520 Journal of Primatology 60 (Supplement 1):62-63

521

522 Faust LJ, Bergstrom YM, Thompson SD, Bier L. 2019. PopLink Version 2.5. Lincoln Park Zoo, 523 Chicago, IL.

524 
525 Gurven M, Kaplan H. 2007. Longevity among hunter-gatherers: a cross-cultural examination.

526 Population and Development Review 33:321-365 DOI: 10.1111/j.1728-4457.2007.00171.x.

527

528 Hämäläinen A, Dammhahn M, Aujard F, Eberle M, Hardy I, Kappeler PM, Perret M, Schliehe-

529 Diecks S, Kraus C. 2014. Senescence or selective disappearance? Age trajectories of body mass 530 in wild and captive populations of a small-bodied primate. Proceedings of the Royal Society B:

531 Biological Sciences 281:20140830 DOI: 10.1098/rspb.2014.0830.

532

533 Havercamp K, Watanuki K, Tomonaga M, Matsuzawa T, Hirata S. 2019. Longevity and

534 mortality of captive chimpanzees in Japan from 1921 to 2018. Primates 60:525-535 DOI:

535 10.1007/s 10329-019-00755-8.

536

537

538

539

540

541

542

543

544 Hirata S, Morimura N, Watanuki K, Ross S. 2020. The establishment of sanctuaries for former

545 laboratory chimpanzees: Challenges, successes, and cross-cultural context. In: Hopper LM, Ross

546 S, ed. Chimpanzees in context: A comparative perspective on chimpanzee behavior, cognition,

547 conservation, and welfare. Chicago: The University of Chicago Press, 208-229.

548

549

550

551

552

553

554

555

556

557

558

Kleinbaum DG, Klein M. 2005. Survival Analysis. New York: Springer Science and Business Media, Inc.

Kooriyama T, Okamoto M, Yoshida T, Nishida T, Tsubota T, Saito A, Tomonaga M, Matsuzawa M, Akari H, Nishimura H, Miyabe-Nishiwaki T. 2013. Epidemiological study of zoonoses derived from humans in captive chimpanzees. Primates 54:89-98 DOI: 10.1007/s10329-012$0320-8$.

559

Lacy RC, Ballou JD, Pollak JP. 2012. PMx: Software Package for Demographic and Genetic Analysis and Management of Pedigreed Populations. Methods in Ecology and Evolution 3:43337 DOI: 10.1111/j.2041-210X.2011.00148.x.

560

561

562

Littleton J. 2005. Fifty years of chimpanzee demography at Taronga Park Zoo. American Journal 563 
564 Matsuzawa T. 2016. SAGA and GAIN for great apes. Primates 57:1-2 DOI: 10.1007/s10329-

565 015-0504-0.

566

567 Miyabe-Nishiwaki T, Kaneko A, Yamanaka A, Maeda N, Suzuki J, Tomonaga M, Matsuzawa T, 568 Muta K, Nishimura R, Yajima I, Eleveld DJ, Absalom AR, Masui K. 2021. Propofol infusions 569 using a human target controlled infusion (TCI) pump in chimpanzees (Pan troglodytes).

570 Scientific Reports 11:1-8 DOI: 10.1038/s41598-020-79914-7.

571

572 Morimura N. 2003. A note on enrichment for spontaneous tool use by chimpanzees (Pan

573 troglodytes). Applied Animal Behaviour Science 82:241-247 DOI: 10.1016/S0168-

574 1591(03)00058-3.

575

576

577

Morimura N, Idani G, Matsuzawa T. 2011. The first chimpanzee sanctuary in Japan: an attempt

578 to care for the "surplus" of biomedical research. American Journal of Primatology 73:226-32

579

580 DOI: 10.1002/ajp.20887.

Morimura N, Ueno Y. 1999. Influences on the feeding behavior of three mammals in the

581 Maruyama Zoo: Bears, elephants, and chimpanzees. Journal of Applied Animal Welfare Science

582 2:169-186 DOI: 10.1207/s15327604jaws0203_1.

583

584

585

Muller MN, Wrangham RW. 2014. Mortality rates among Kanyawara chimpanzees. Journal of Human Evolution 66:107-114 DOI: 10.1016/j.jhevol.2013.10.004.

586

587

588

Naples LM, Langan JN, Kearns KS. 2010. Comparison of the anesthetic effects of oral

589 transmucosal versus injectable medetomidine in combination with Tiletamine-Zolazepam for immobilization of chimpanzees (Pan troglodytes). Journal of Zoo and Wildlife Medicine 41:50-

590

591

592

593 62 DOI: $10.1638 / 2009-0096.1$.

594

595

596 Ochiai T, Watanuki K, Udono T, Morimura N, Hirata S, Tomonaga M, Idani G, Matsuzawa T.

597 2015. The history of captive chimpanzees (Pan troglodytes) in Japan 1920-1950. Primate

598

Research 31:19-29 DOI: 10.2354/psj.31.001.

599

600 R Core Team. 2020. R: A language and environment for statistical computing, version 3.6.2. R

601 Foundation for Statistical Computing, Vienna, Austria.

602 
603 Ross SR, Bloomsmith MA, Bettinger TL, Wagner KE. 2009. The influence of captive adolescent 604 male chimpanzees on wounding: management and welfare implications. Zoo Biology 28:623-634 605 DOI: 10.1002/zoo.20243.

606

607 Ross S. 2020. Chimpanzee (Pan troglodytes) AZA Regional Chimpanzee Studbook (current to 608 2/1/2020). Lincoln Park Zoo, Chicago, IL.

609

610 Saiyed ST, Liubicich RC, Fidino M, Ross SR. 2018. Stillbirth rates across three ape species in 611 accredited American zoos. American Journal of Primatology 80:e22870 DOI:

612 10.1002/ajp.22870.

613

614 Seifarth JE, McGowan CL, Milne KJ. 2012. Sex and life expectancy. Gender Medicine 9:390615 401 DOI: 10.1016/j.genm.2012.10.001.

616

617 Seres M, Aureli F, de Waal FB. 2001. Successful formation of a large chimpanzee group out of 618 two preexisting subgroups. Zoo Biology 20:501-515 DOI: 10.1002/zoo.10003.

619

620

Struck K, Videan EN, Fritz J, Murphy J. 2007. Attempting to reduce regurgitation and

621 reingestion in a captive chimpanzee through increased feeding opportunities: a case study. Lab

622 Animal 36:35-38 DOI: 10.1038/laban0107-35.

623

624 Therneau T. 2021. A package for survival analysis in R. R package version 3.2-11, 625 https://CRAN.R-project.org/package=survival.

626

627 Thompson ME, Jones JH, Pusey AE, Brewer-Marsden S, Goodall J, Marsden D, Matsuzawa T,

628 Nishida T, Reynolds V, Sugiyama Y, Wrangham RW. 2007. Aging and fertility patterns in wild chimpanzees provide insights into the evolution of menopause. Current Biology 17:2150-2156

630 DOI: 10.1016/j.cub.2007.11.033.

631

632 Tidière M, Gaillard JM, Berger V, Müller DW, Lackey LB, Gimenez O, Clauss M, Lemaître JF. 633 2016. Comparative analyses of longevity and senescence reveal variable survival benefits of 634 living in zoos across mammals. Scientific Reports 6:36361 DOI: 10.1038/srep36361.

635

636 Vaupel JW, Manton KG, Stallard E. 1979. The impact of heterogeneity in individual frailty on 637 638

639 the dynamics of mortality. Demography 16:439-454 DOI: 10.2307/2061224. Vaupel JW, Yashin AI. 1985. Heterogeneity's ruses: some surprising effects of selection on population dynamics. The American Statistician 39:176-185. 
642 Vina J, Gambini J, Lopez-Grueso R, M Abdelaziz K, Jove M, Borras C. 2011. Females live 643 longer than males: role of oxidative stress. Current Pharmaceutical Design 17:3959-3965 DOI:

$64410.2174 / 138161211798764942$.

645

646 Watanuki K, Ochiai T, Hirata S, Morimura N, Tomonaga M, Idani G, Matsuzawa T. 2014.

647 Review and long-term survey of the status of captive chimpanzees in Japan in 1926-2013.

648 Primate Research 30:147-156 DOI: 10.2354/psj.30.009.

649

650 Wiese RJ, Willis K. 2004. Calculation of longevity and life expectancy in captive elephants. Zoo 651 Biology 23:365-373 DOI: 10.1002/zoo.20011.

652

653 Williams RC, Nash LT, Scarry CJ, Videan EN, Fritz J. 2010. Factors affecting wounding 654 aggression in a colony of captive chimpanzees (Pan troglodytes). Zoo Biology 29:351-364 DOI: 655 10.1002/zoo.20263.

656

657 Wood BM, Watts DP, Mitani JC, Langergraber KE. 2017. Favorable ecological circumstances 658 promote life expectancy in chimpanzees similar to that of human hunter-gatherers. Journal of 659 Human Evolution 105:41-56 DOI: 10.1016/j.jhevol.2017.01.003.

660

661 Yamanashi Y, Morimura N, Mori Y, Hayashi M, Suzuki J. 2013. Cortisol analysis of hair of 662 captive chimpanzees (Pan troglodytes). General and Comparative Endocrinology 194:55-63 663 DOI: 10.1016/j.ygcen.2013.08.013. 
Figure 1

Age-specific survival rates for females and males, from life tables for a wild population and the AZA and Japan captive populations of chimpanzees from birth (top) and from age 1 (bottom).

Wild population data is from Gombe, Tanzania (Bronikowski et al. 2016). 

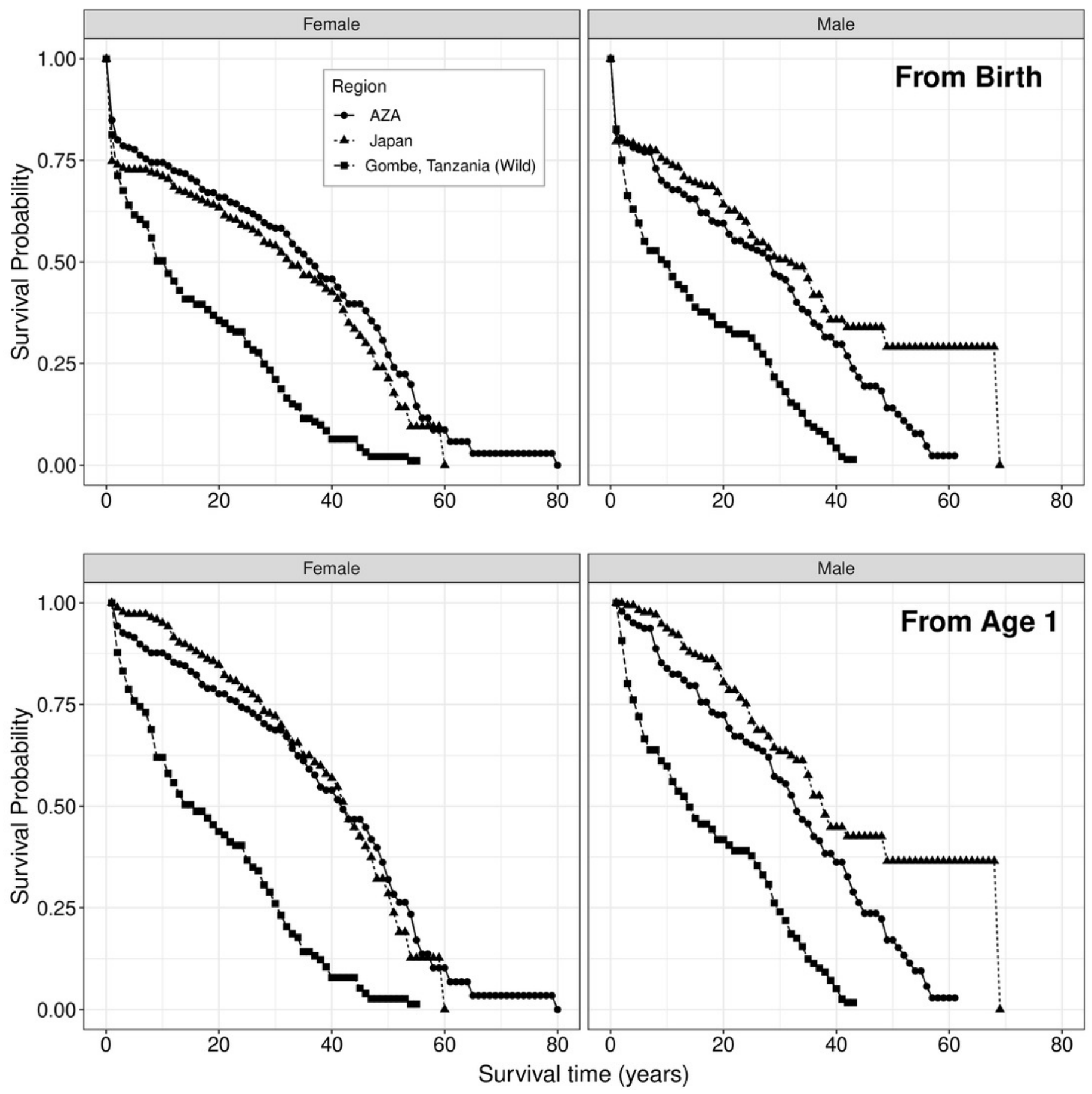


\section{Figure 2}

Observed distribution of ages at death by sex, time period, and birth type in (A) the AZA and (B) the Japan captive chimpanzee populations.

Only deaths occurring on or after age one are included. The "early" time period is 1975-2000 for AZA and 1980-2000 for Japan, and the "recent" period is 2001-2020 for both. Sample sizes for the number of observed deaths for females and males are included in parentheses for each group. Note that the $y$-axis range differs between panels. 

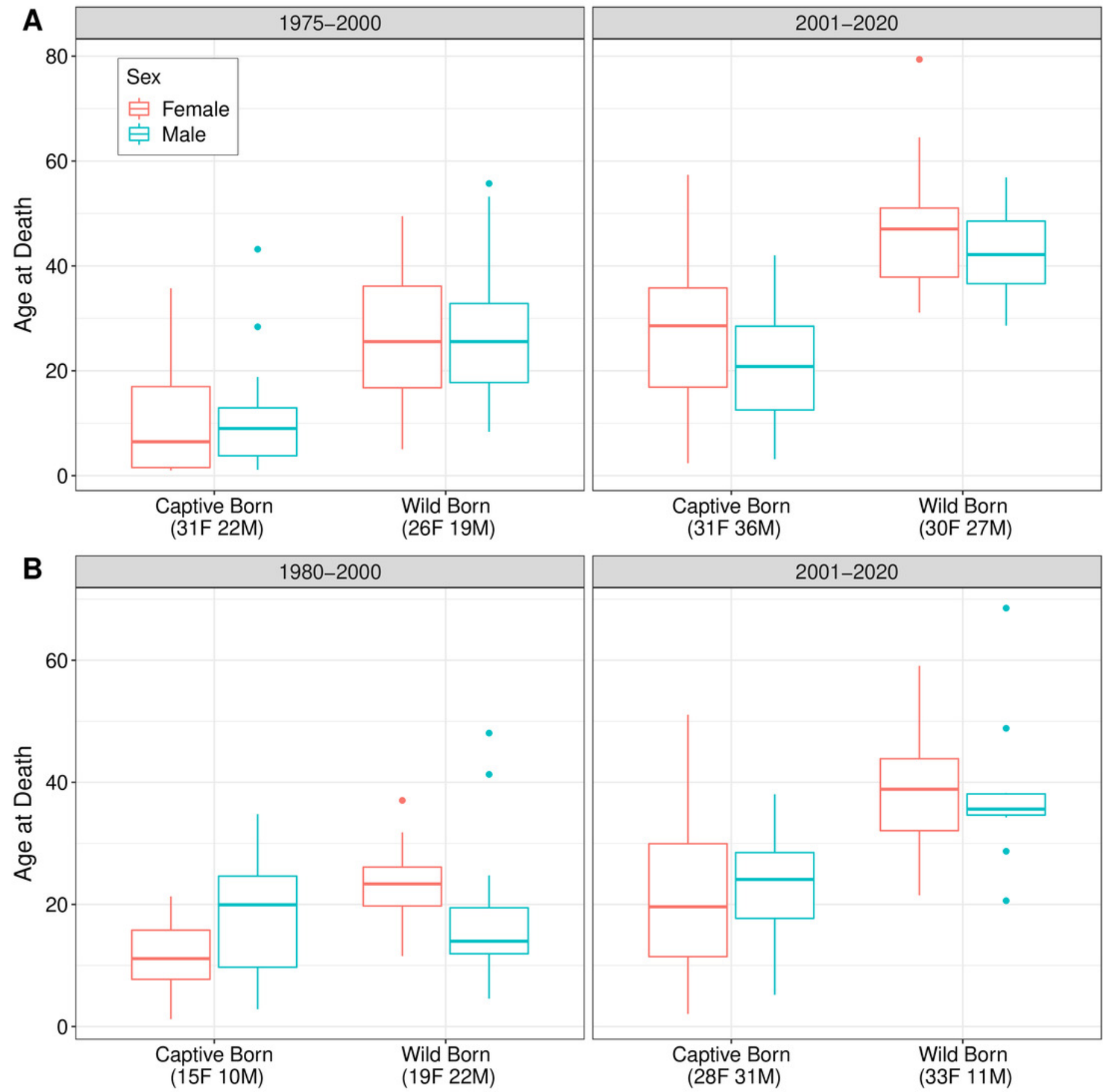


\section{Figure 3}

Median age and total population size of the AZA and Japan captive chimpanzee populations over time.

Ages were tabulated starting at age one and censused at Dec. 31 of each year (except for the last year, which was censused on the last data compilation date of 2/1/2020). Individuals who had not yet reached age one or had an unknown birth location (30 individuals in the Japan population and 6 in the AZA population as of Feb. 2020) were not included in this figure. 


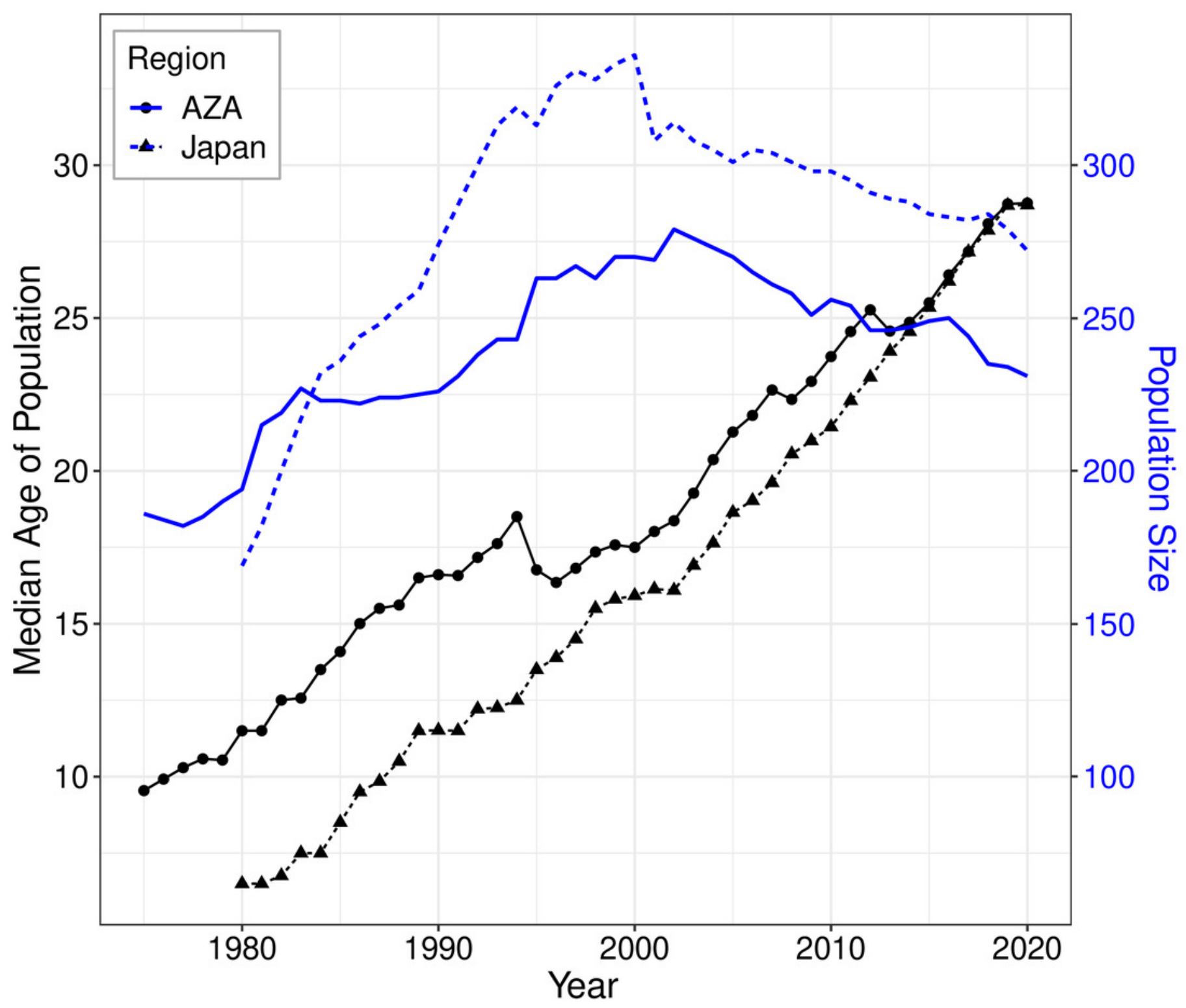


Figure 4

Numbers of deaths in the AZA (top panel) and the Japan (bottom panel) captive chimpanzee populations by month (AZA: 1975-2020, Japan: 1980-2020).

Seasons are grouped in alternating colors: winter $=$ Dec-Feb (green); spring = Mar-May (gray); summer = Jun-Aug (green); Fall = Sep-Nov (gray). Darker bars include all observed deaths, and lighter bars include only deaths on or after age one. 

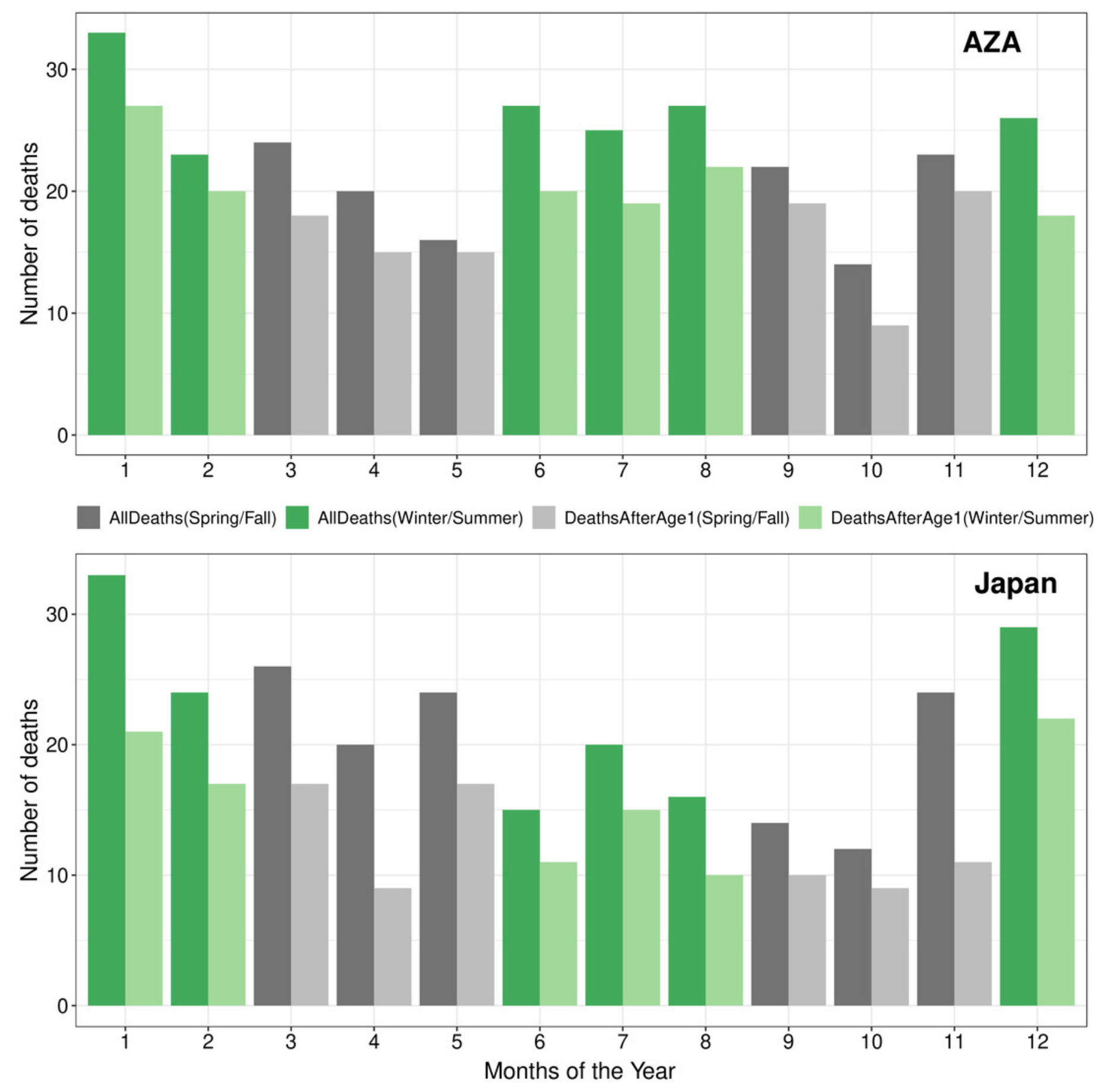


\section{Table $\mathbf{1}$ (on next page)}

Summary of existing studies describing wild or captive chimpanzee life history patterns.

When full life tables are presented for both females and males, life expectancy estimates are combined. 


\begin{tabular}{|c|c|c|c|c|c|}
\hline Source & $\begin{array}{l}\text { Population } \\
\text { (captive or } \\
\text { wild) }\end{array}$ & Location & Sample size & $\begin{array}{l}\text { Life expectancy } \\
\text { of live born } \\
\text { individuals }\end{array}$ & $\begin{array}{l}\text { Life expectancy of } \\
\text { individuals who } \\
\text { survived beyond } 1 \\
\text { year (calculated from } \\
\text { life table) }\end{array}$ \\
\hline $\begin{array}{l}\text { Havercamp } \\
\text { et al. } 2019\end{array}$ & Captive & $\begin{array}{l}\text { Japan } \\
1921-2018\end{array}$ & 821 & $\begin{array}{l}\text { Both }(\text { sexes })= \\
28.3 y\end{array}$ & $\begin{array}{l}\text { From } 1 y=34.6 y \\
\text { From } 12 y=40.4 y \\
\text { From } 15 y=42.4 y\end{array}$ \\
\hline $\begin{array}{l}\text { Littleton } \\
2005\end{array}$ & Captive & $\begin{array}{l}\text { Australia } \\
\text { 1941-2000 }\end{array}$ & 113 & $\begin{array}{l}\text { Not presented, } \\
\text { no life table }\end{array}$ & Not presented \\
\hline $\begin{array}{l}\text { Dyke et al. } \\
1995\end{array}$ & Captive & $\begin{array}{l}3 \text { breeding } \\
\text { colonies in the } \\
\text { United States } \\
\text { (years unknown) }\end{array}$ & $\begin{array}{l}\mathrm{F}=650 \\
(\text { Total } \mathrm{N}= \\
1,346, \text { but } \mathrm{e}_{\mathrm{x}} \\
\text { not provided } \\
\text { for males) }\end{array}$ & $\begin{array}{l}\text { Females only } \\
\text { (observed data) }= \\
23.1 \mathrm{y} \text {, not reliable } \\
\text { due to young } \\
\text { population at the } \\
\text { time }\end{array}$ & $\begin{array}{l}\text { Not presented - } \\
\text { Only model life tables } \\
\text { presented }\end{array}$ \\
\hline $\begin{array}{l}\text { Courtney \& } \\
\text { Santow } 1989\end{array}$ & Captive & $\begin{array}{l}\text { Australia \& New } \\
\text { Zealand } \\
1935-1983\end{array}$ & 87 & $\begin{array}{l}\text { Not possible to } \\
\text { calculate late life } \\
\text { expectancy due to } \\
\text { small, young } \\
\text { population } \\
\text { (mortality } \\
\text { probabilities up to } \\
\text { 30y presented) }\end{array}$ & Not presented \\
\hline $\begin{array}{l}\text { Wood et al. } \\
2017\end{array}$ & Wild & $\begin{array}{l}\text { Ngogo, Uganda } \\
1995-2016\end{array}$ & 306 & Both $=32.8 \mathrm{y}$ & $\begin{array}{l}\text { From } 1 y=38.6 y \\
\text { From } 12 y=43.1 y \\
\text { From } 15 y=43.1 y\end{array}$ \\
\hline $\begin{array}{l}\text { Bronikowski } \\
\text { et al. } 2016\end{array}$ & Wild & $\begin{array}{l}\text { Gombe, Tanzania } \\
1963-2013\end{array}$ & $\begin{array}{l}\mathrm{F}=155 \\
(\text { Total } \mathrm{N}=288, \\
\text { but } \mathrm{e}_{\mathrm{x}} \text { not } \\
\text { provided for } \\
\text { males) }\end{array}$ & $\begin{array}{l}\text { Females only }= \\
15.9 \mathrm{y}\end{array}$ & $\begin{array}{l}\text { Females only }= \\
\text { From } 1 \mathrm{y}=19.4 \mathrm{y} \\
\text { From } 12 \mathrm{y}=30.2 \mathrm{y} \\
\text { From } 15 \mathrm{y}=32.0 \mathrm{y}\end{array}$ \\
\hline $\begin{array}{l}\text { Bronikowski } \\
\text { et al. } 2011\end{array}$ & Wild & $\begin{array}{l}\text { Gombe, Tanzania } \\
1963-2008\end{array}$ & $\begin{array}{l}F=144 \\
M=122\end{array}$ & $\begin{array}{l}\text { Females }=16 y \\
\text { (range: } 10-25 y \text { ) } \\
\text { Males }=11 \mathrm{y} \\
\text { (range: } 9-14 y \text { ) }\end{array}$ & Not presented \\
\hline $\begin{array}{l}\text { Muller \& } \\
\text { Wrangham } \\
2014\end{array}$ & Wild & $\begin{array}{l}\text { Kanyawara, } \\
\text { Uganda } \\
1989-2013\end{array}$ & 123 & Both $=19.4 \mathrm{y}$ & $\begin{array}{l}\text { From } 1 y=22 y \\
\text { From } 12 y=34.5 y \\
\text { From } 15 y=39.2 y\end{array}$ \\
\hline
\end{tabular}




\begin{tabular}{|c|c|c|c|c|c|}
\hline $\begin{array}{l}\text { Hill et al. } \\
2001\end{array}$ & Wild & $\begin{array}{l}5 \text { study } \\
\text { populations } \\
\text { Gombe, Tanzania: } \\
\text { 1963-1998; } \\
\text { Taï, Ivory Coast: } \\
\text { 1982-1994; } \\
\text { Kanyawara, } \\
\text { Uganda: 1989- } \\
\text { 1998; } \\
\text { Mahale, Tanzania: } \\
\text { K group 1966- } \\
\text { 1988, M group } \\
\text { 1979-1988; } \\
\text { Bossou, Guinea: } \\
\text { 1976-1993 }\end{array}$ & $\begin{array}{l}179+ \\
123+ \\
74+ \\
92+ \\
22 \\
=490\end{array}$ & Both $=12.9 \mathrm{y}$ & $\begin{array}{l}\text { From } 1 \mathrm{y}=15.6 \mathrm{y} \\
\text { From } 12 \mathrm{y}=27.4 \mathrm{y} \\
\text { From } 15 \mathrm{y}=29.8 \mathrm{y}\end{array}$ \\
\hline
\end{tabular}

2 


\section{Table 2 (on next page)}

Kaplan-Meier median life expectancy (MLE) estimates for the AZA population (1975-2020), the Japan population (1980-2020), and both populations of captive chimpanzees combined.

Separate estimates are presented for females and males and starting from birth or from age one (i.e., assuming survival to first birthday), with their $95 \%$ confidence intervals in parentheses. For the Japan population male estimate, the upper limit was undefined because many older males are still living. 


\section{Female MLE Male MLE}

\section{From birth}

$\begin{array}{lll}\text { AZA } & 36.7(32.7-42.1) & 28.4(21.7-32.5) \\ \text { Japan } & 34.1(30.2-41.2) & 34.0(27.1-38.0) \\ \text { AZA + Japan } & 35.7(32.4-40.0) & 30.1(27.3-34.3)\end{array}$

From age one

$\begin{array}{lll}\text { AZA } & 41.9(37.4-47.5) & 32.5(29.4-37.5) \\ \text { Japan } & 42.4(39.9-47.2) & 37.7(34.8-N A) \\ \text { AZA + Japan } & 42.4(40.0-46.3) & 35.5(32.6-38.0)\end{array}$

1 


\section{Table 3(on next page)}

Results from Poisson regressions comparing whether the median age at mortality in the AZA and Japan populations differed based on sex, birth type, and between two time periods in captive chimpanzees.

The "early" time period is 1975-2000 for AZA and 1980-2000 for Japan, the "recent" period is 2001-2020 for both. Analyses were performed using only mortalities that occurred on or after the first birthdate. Bolded coefficient estimates highlight the effects that were significant at $p<0.05$. 
AZA

(Intercept)

Sex (Male)

Period (Late)

Birth type (Wild)

Sex (Male) * Period (Late)

Period (Late) * Birth type (Wild)

Sex (Male) * Birth type (Wild)

Null deviance: 2528.3 on 221 degrees of freedom

Residual deviance: 1255.8 on 215 degrees of freedom

Dispersion parameter for quasipoisson family taken to be 5.782681

1
Japan

$\begin{array}{rrrrl} & \text { Std. } & & & \\ \text { Estimate } & \text { Error } & \text { z value } & \text { p value } & \\ \mathbf{2 . 4 1} & 0.12 & 20.75 & <2 \mathrm{E}-16 & \text { (Intercept) } \\ -0.08 & 0.15 & -0.53 & 0.5951 & \text { Sex (Male) } \\ \mathbf{0 . 8 9} & 0.13 & 6.82 & 9.33 \mathrm{E}-11 & \text { Period (Late) } \\ \mathbf{0 . 8 7} & 0.13 & 6.50 & 5.52 \mathrm{E}-10 & \text { Birth type (Wild) } \\ -0.16 & 0.14 & -1.18 & 0.2407 & \text { Sex (Male) * Period (Late) } \\ \mathbf{- 0 . 3 2} & 0.14 & -2.19 & 0.0297 & \text { Sex (Male) * Birth type (Wild) } \\ 0.13 & 0.13 & 0.97 & 0.3319 & \text { Period (Late) * Birth type (Wild) } \\ & & & & \text { Sex * Birth type * Period }\end{array}$

* Period

Null deviance: 1203.96 on 168 degrees of freedom

Residual deviance: 686.09 on 161 degrees of freedom

Dispersion parameter for quasipoisson family taken to be 4.176631 